\title{
Symbolic dynamics and chaotic synchronization
}

\author{
Acilina Caneco $^{(1)}$, Clara Grácio ${ }^{(2)}$ and J. Leonel Rocha ${ }^{(3)}$
}

(1) Mathematics Unit, Instituto Superior de Engenharia de Lisboa, Lisboa and CIMA-UE Évora, Portugal. Email: acilina@deetc.isel.ipl.pt. (2) Department of Mathematics, Universidade de Évora and CIMA-UE, Évora, Portugal. Email: mgracio@uevora.pt (3) Mathematics Unit, Instituto Superior de Engenharia de Lisboa and CEAUL, Lisboa, Portugal. Email: irocha@deq.isel.ipl.pt

\begin{abstract}
Chaotic communications schemes based on synchronization aim to provide security over the conventional communication schemes. Symbolic dynamics based on synchronization methods has provided high quality synchronization [5]. Symbolic dynamics is a rigorous way to investigate chaotic behavior with finite precision and can be used combined with information theory [13]. In previous works we have studied the kneading theory analysis of the Duffing equation [3] and the symbolic dynamics and chaotic synchronization in coupled Duffing oscillators [2] and [4]. In this work we consider the complete synchronization of two identical coupled unimodal and bimodal maps. We relate the synchronization with the symbolic dynamics, namely, defining a distance between the kneading sequences generated by the map iterates in its critical points and defining $n$-symbolic synchronization. We establish the synchronization in terms of the topological entropy of two unidirectional or bidirectional coupled piecewise linear unimodal and bimodal maps. We also give numerical simulations with coupled Duffing oscillators that exhibit numerical evidence of the $n$-symbolic synchronization.

Keywords: Chaotic synchronization, Symbolic dynamics, Symbolic synchronization, Kneading theory.
\end{abstract}

\section{Introduction}

Synchronization of two or more systems, is a process wherein the systems adjust a given property of their motion to a common behaviour, due to coupling or forcing. Chaos synchronization is the agreement or correlation of different chaotic processes in time. A great deal of researches has been undertaken on this subject, since the pioneering works of Pecora and Carroll ([9], [10]). Various types of synchronization have been studied. This includes complete synchronization, phase synchronization, lag synchronization, generalized synchronization, anticipated synchronization, and so on [1]. The coupled systems might be identical or different, the coupling might be unidirectional, (master-slave or drive-response), or bidirectional (mutual coupling) and the driving force might be deterministic or stochastic [11].

\footnotetext{
Chaos Theory: Modeling, Simulation and Applications

C. H. Skiadas, I. Dimotikalis and C. Skiadas (Eds)

(C) 2011 World Scientific Publishing Co. (pp. 135 - 142)
} 
In this paper we relate the synchronization with symbolic dynamics, namely, defining a distance between the kneading sequences generated by the map iterates in its critical points and defining $n$-symbolic synchronization. We will see that this distance is a good measure of synchronization, since it decreases to zero, as the coupling parameter increases and the oscillators begin to synchronize. Moreover we establish in two theorems the synchronization in terms of the topological entropy of two unidirectional or bidirectional coupled piecewise linear unimodal and bimodal maps. The $n$ symbolic synchronization is numerically showed for Duffing oscillators.

\section{The Synchronization Problem}

Consider two coupled systems $x_{n+1}=f\left(x_{n}\right)$ and $y_{n+1}=f\left(y_{n}\right)$. The two identical systems are in complete synchronization, if the difference $z_{n}=y_{n}-x_{n}$ converges to zero, as $n \rightarrow \infty$. The coupling may be unidirectional

$$
\begin{aligned}
& \left\{\begin{array}{c}
x_{n+1}=f\left(x_{n}\right) \\
y_{n+1}=f\left(y_{n}\right)+k\left[f\left(x_{n}\right)-\left(y_{n}\right)\right]
\end{array}\right. \\
& z_{n+1}=(1-k)\left[f\left(y_{n}\right)-f\left(x_{n}\right)\right],
\end{aligned}
$$

or bidirectional

$$
\begin{gathered}
\left\{\begin{array}{l}
x_{n+1}=f\left(x_{n}\right)-k\left[f\left(x_{n}\right)-\left(y_{n}\right)\right] \\
y_{n+1}=f\left(y_{n}\right)+k\left[f\left(x_{n}\right)-\left(y_{n}\right)\right]
\end{array}\right. \\
z_{n+1}=(1-2 k)\left[f\left(y_{n}\right)-f\left(x_{n}\right)\right],
\end{gathered}
$$

where $k$ is the coupling parameter. In [9] and [10] it is establish that this kind of synchronization can be achieved provided that all the Lyapunov exponents are negative.

Motivated by the fact that any transitive $m$-modal map with positive topological entropy and growth number $\pm s$, is topologically semiconjugated to a piecewise linear map with slope $\pm s$ everywhere, see [8], we will study the synchronization of unimodal and bimodal piecewise linear maps.

\subsection{Coupling two unimodal piecewise linear maps}

Consider the tent map $f_{s}:[0,1] \rightarrow[0,1]$ defined by $f_{s}(x)=s x-s+2$,

if $0 \leq x \leq 1-1 / s$ and $f_{s}(x)=s-s x$, if $1-1 / s \leq x \leq 1$. This map $f_{s}$ can be written as

$$
f_{s}(x)=s x-s+2+2 \theta(x)(s-s x-1)
$$

with $\theta(x)=1$, if $0 \leq x \leq 1-1 / s$ and $\theta(x)=0$, if $1-1 / s \leq x \leq 1$. 
Note that, there exist a one-to-one correspondence between the images of the $\operatorname{map} \theta,\{0,1\}$, and the usual alphabet $A=\{L, R\}$, associated to the unimodal map. With the kneading theory we construct symbolic sequences associated with the critical points of the map, see [3], [7] and [8]. Noticing that, as the coupled systems became synchronized, the number of equal initial symbols in the kneading sequences grows (see Table 1), we are led to the following.

Definition 2.1. Let $S_{z}=S_{z_{1}} S_{z_{2}} \ldots S_{z_{n}} \ldots$, be a symbolic sequence, associated to the orbit of a point $z$ of the "map $f$, using in position $i$ a symbol $S_{z}$ belonging to some alphabet $A$. Define a distance between two sequences

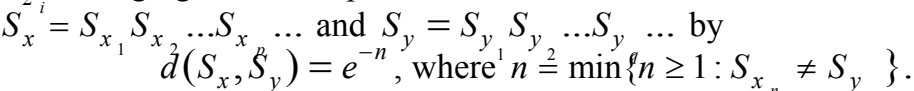

Definition 2.2. In the conditions of the previous definition, "two sequences $S_{x}=S_{x_{1}} S_{x_{2}} \ldots S_{x_{n}} \ldots$ and $S_{y}=S_{y_{1}} S_{y_{2}} \ldots S_{y_{q}} \ldots$ are said $n$-symbolic synchronized iff $\exists n \in \mathrm{N}: d\left(S_{x}, S_{y}\right) \leq e^{-n_{2}}$.

Note that $d\left(S_{x}, S_{y}\right)=e^{-n}$, means that, $S_{x_{i}}=S_{y} \quad(1 \leq i<n)$ and $n=\min \left\{n \geq 1: S_{x} \neq S_{y}\right\}$. For the unimodal map $f_{s}$, we used $\theta(x)$ to represent the symbolic sequence $S_{x}$. In this case, the symbol $S_{x_{i}}=\theta\left(x_{i}\right)$, in the position $i$ of $S_{x}$, can only be 0 or 1 . As we have $x_{n+1}=f\left(x_{n}\right)$, we must consider $\theta\left(x_{n}\right)$ and $\theta\left(x_{n+1}\right)$.

Theorem 2.1. Let $x_{n+1}=f\left(x_{n}\right)$ and $y_{n+1}=f\left(y_{n}\right)$ be two identical coupled systems, with $f$ given by (3) and $1<s \leq 2$. Let $h$ be the topological entropy of (3) $(h=\log s)$ and $k \in[0,1]$ the coupling parameter. If $\theta(y)$ and $\theta(x)$ are $n$-symbolic synchronized, then

1) The unidirectional coupled systems (1) are synchronized if $k>1-e^{-h}$.

2) The bidirectional coupled systems (2) are synchronized if

$$
(1 / 2)\left(1-e^{-h}\right)<k<(1 / 2)\left(1+e^{-h}\right) .
$$

Proof. Attending to (1) and (3), we have for the unidirectional case $z_{n+1}=(1-k)\left[s y_{n}+2 \theta\left(y_{n}\right)\left(s-s y_{n}-1\right)-s x_{n}-2 \theta\left(x_{n}\right)\left(s-s x_{n}-1\right)\right]$. From the considered hypothesis, it follows that, for $n$ sufficiently large, $\theta\left(y_{n}\right)=\theta\left(x_{n}\right)=\theta$, and $z_{n+1}=(1-k)(1-2 \theta) s z_{n}$.

Then, $z_{n+m}=[(1-k)(1-2 \theta) s]^{m} z_{n}$. We may writte $z_{n+m}=q^{m} z_{n}$, with $q=(1-k)(1-2 \theta) s$. So, we have $\lim q^{m}=0$, iff $|q|<1$. From the definition of $\theta,|1-2 \theta|=1$, then, to have complete synchronization of the unidirectionally coupled maps it suffices $k>1-s^{-1}$, i.e., $k>1-e^{-h}$.

On the other hand, attending to (2) and (3), we have for the bidirectional case $z_{n+1}=(1-2 k)\left[s y_{n}+2 \theta\left(y_{n}\right)\left(s-s y_{n}-1\right)-s x_{n}-2 \theta\left(x_{n}\right)\left(s-s x_{n}-1\right)\right]$. From the considered hypothesis, it follows that, for $n$ large enough,

$\theta\left(y_{n}\right)=\theta\left(x_{n}\right)=\theta$, and $z_{n+1}=(1-2 k)(1-2 \theta) s z_{n}$.

Then, $z_{n+m}=[(1-2 k)(1-2 \theta) s]^{m} z_{n}$. So, we have $\lim z_{n+m}=0$, iff $|1-2 k||1-2 \theta| s<1$. From the definition of $\theta,|1-2 \theta|_{=}^{m} \rightarrow^{\infty}$, then, to have complete synchronization of the bidirectionally coupled maps it suffices (4). 


\subsection{Coupling two bimodal piecewise linear maps}

Consider the bimodal piecewise linear map $f_{s, r}:[0,1] \rightarrow[0,1]$, with slopes $\pm s$, defined by

$$
f_{s, r}=\left\{\begin{array}{ccc}
-s x+1 & \text { if } & 0 \leq x<c_{1} \\
s x+r-1 & \text { if } & c_{1} \leq x<c_{2} \\
-s x+s & \text { if } & c_{2} \leq x \leq 1
\end{array}\right.
$$

with $r=(3+s) / 2-s\left(c_{1}+c_{2}\right)$ and critical points $c_{1}=(2-r) / 2 s$ and $c_{2}=(1+s-r) / 2 s$, see [7]. Recall that any transitive bimodal map is topologically semi-cojugated to such a map. The map $f_{s, r}$ can be written as

$$
f_{s, r}(x)=-s x+1+\theta_{c_{1}}(x)(2 s x+r-2)+\theta_{c_{2}}(y)(-2 s x+s-r-1),
$$

with

$$
\theta_{c_{i}}(x)=\left\{\begin{array}{lll}
0, & \text { if } & 0 \leq x<c_{i} \\
1, & \text { if } & c_{i} \leq x \leq 1
\end{array} \quad(i=1,2) .\right.
$$

In this case, we may define $\theta(x)=\theta_{c_{1}}(x)+\theta_{c_{2}}(x)$, i.e.,

$$
\theta(x)=\left\{\begin{array}{lll}
0 & \text { if } & 0 \leq x<c_{1} \\
1 & \text { if } & c_{1} \leq x<c_{2} \\
2 & \text { if } & c_{2} \leq x \leq 1
\end{array}\right.
$$

Note the existence of a one-to-one correspondence between the images of the $\operatorname{map} \theta,\{0,1,2\}$, and the usual alphabet associated to the bimodal map $A=\{L$, $M, R\}$, see [3], [8] and [6]. For the bimodal map $f_{s, r}$, the symbol $S_{x_{i}}=\theta\left(x_{i}\right)$, in the position $i$ of $S_{x}=\theta(x)$, can be 0,1 , or 2 . Now, we can state a theorem for bimodal maps similar to the one to unimodal maps. The conclusions are the same, although for a different local map $f$.

Theorem 2.2. Let $x_{n+1}=f\left(x_{n}\right)$ and $y_{n+1}=f\left(y_{n}\right)$ be two identical coupled systems, with $f$ given by (5) and $1<s \leq 2$. Let $h$ be be the topological entropy of (5) $(h=\log s)$ and $k \in[0,1]$ the coupling parameter. If $\theta_{c_{i}}(x)$ and $\theta_{c_{i}}(y)$ for $i=1,2$, are $n$-symbolic synchronized, then

1) The unidirectional coupled systems (1) are synchronized if $k>1-e^{-h}$.

2) The bidirectional coupled systems (2) are synchronized if

$$
(1 / 2)\left(1-e^{-h}\right)<k<(1 / 2)\left(1+e^{-h}\right)
$$

Prof. Attending to (1) and (5), we have for the unidirectional case

$$
\begin{aligned}
z_{n+1}= & (1-k)\left\{\left[-s y_{n}+1+\theta_{c_{1}}\left(y_{n}\right)\left(2 s y_{n}+r-2\right)+\theta_{c_{2}}\left(y_{n}\right)\left(-2 s y_{n}+s-r+1\right)\right]\right. \\
& \left.-\left[-s x_{n}+1+\theta_{c_{1}}\left(x_{n}\right)\left(2 s x_{n}+r-2\right)+\theta_{c_{2}}\left(x_{n}\right)\left(-2 s x_{n}+s-r+1\right)\right]\right\} .
\end{aligned}
$$

From the considered hypothesis, it follows that, for $n$ large enough, 


$$
\begin{gathered}
\theta_{c_{1}}\left(y_{n}\right)=\theta_{c_{1}}\left(x_{n}\right)=\theta_{c_{1}} \text { and } \theta_{c_{2}}\left(y_{n}\right)=\theta_{c_{2}}\left(x_{n}\right)=\theta_{c_{2}} \text { and } \\
z_{n+1}=(1-k)\left[-1-2\left(\theta_{c_{1}}-\theta_{c_{2}}\right)\right] s z_{n} .
\end{gathered}
$$

Then, $\quad z_{n+m}=\left[(1-k)\left[-1-2\left(\theta_{c_{1}}-\theta_{c_{2}}\right)\right] s\right]^{m} z_{n}$. We may writte $z_{n+m}=q^{m} z_{n}$, with $\quad q=(1-k)\left[-1-2\left(\theta_{c_{1}}-\theta_{c_{2}}\right)\right] s . \quad$ So, we have $\lim _{m \rightarrow \infty} z_{n+m}=0$, iff $|q|=|1-k|\left|-1-2\left(\theta_{c_{1}}-\theta_{c_{2}}\right)\right| s<1$. Attending that,

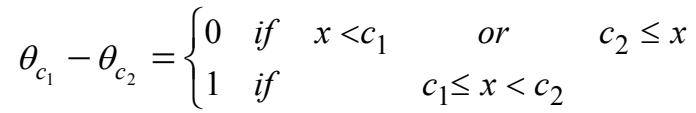

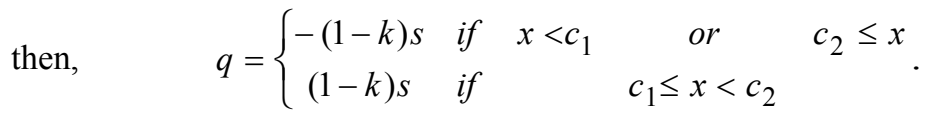

So, $|q|=(1-k) s$, and to have complete synchronization it suffices, $k>1-s^{-1}$, i.e., $k>1-e^{-h}$.

For the bidirectional case and attending to (2) and (5), we have

$$
\begin{aligned}
z_{n+1}= & (1-2 k)\left\{\left[-s y_{n}+1+\theta_{c_{1}}\left(y_{n}\right)\left(2 s y_{n}+r-2\right)+\theta_{c_{2}}\left(y_{n}\right)\left(-2 s y_{n}+s-r+1\right)\right]\right. \\
& \left.-\left[-s x_{n}+1+\theta_{c_{1}}\left(x_{n}\right)\left(2 s x_{n}+r-2\right)+\theta_{c_{2}}\left(x_{n}\right)\left(-2 s x_{n}+s-r+1\right)\right]\right\} .
\end{aligned}
$$

From the hypothesis, $\theta_{c_{1}}\left(y_{n}\right)=\theta_{c_{1}}\left(x_{n}\right)=\theta_{c_{1}}$ and $\theta_{c_{2}}\left(y_{n}\right)=\theta_{c_{2}}\left(x_{n}\right)=\theta_{c_{2}}$, for $n$ large enough. Then, $z_{n+m}=\left[(1-2 k)\left[-1+2\left(\theta_{c_{1}}-\theta_{c_{2}}\right)\right] s\right]^{m} z_{n}$. So, $\lim _{m \rightarrow \infty} z_{n+m}=0$, iff $\left|1-2 k \|-1+2\left(\theta_{c_{1}}-\theta_{c_{2}}\right)\right| s<1$. As in the previous case, $\left|-1+2\left(\theta_{c_{1}}-\theta_{c_{2}}\right)\right|=1$, so the complete synchronization of the bidirectionally coupled maps happens if (6) is verified.

\section{Duffing application}

Consider two identical bidirectionally coupled Duffing oscillators, [12].

$$
\left\{\begin{array}{l}
x^{\prime \prime}(t)=x(t)-x^{3}(t)-\alpha x^{\prime}(t)+k[y(t)-x(t)]+\beta \operatorname{Cos}(w t) \\
y^{\prime \prime}(t)=y(t)-y^{3}(t)-\alpha y^{\prime}(t)-k[y(t)-x(t)]+\beta \operatorname{Cos}(w t)
\end{array} .\right.
$$

We consider parameter values for which each uncoupled $(\mathrm{k}=0)$ oscillator exhibits a chaotic behavior, so if they synchronize, that will be a chaotic synchronization. A basic tool is to do an appropriate Poincaré section. In our case, we did a section defined by $y=0$, since it is transversal to the flow, it contains all fixed points and captures most of the interesting dynamics. In a previous work we have found in the parameter plane $(\alpha, \beta)$ regions $U$ and $B$ where the first return Poincaré map behaves like a unimodal map and like a bimodal map respectively, see [4]. 


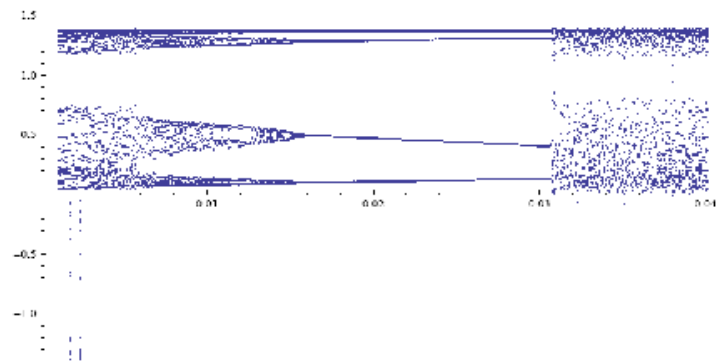

Figure 1. Bifurcation diagram for $x_{n}$ as a function of $k \in[0.001,0.04]$, and parameter values, in the unimodal region.
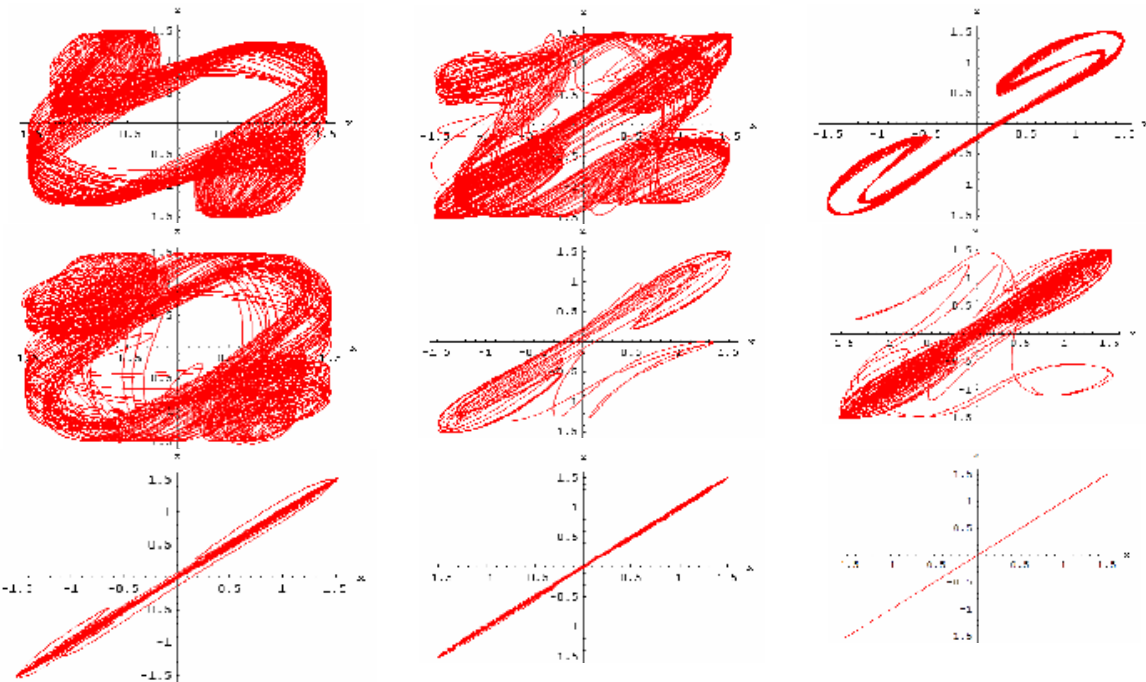

Figure 2. $x$ versus $y$ in the bimodal case for $(\alpha, \beta)=(0.5,0.719)$ for increasing $k$.

Fig. 1 shows the bifurcation diagram for $x_{n}$ as a function of $k \in[0.001,0.04]$, for parameter values for which the Poincaré map behaves like a unimodal.

We computed the kneading sequences, the kneading determinant and the topological entropy for some values of the parameters $(\alpha, \beta)$, see [2] and [4]. The synchronization will occur when $x=y$. See in Fig.2 the evolution of $x$ versus $y$ for $k=0.008,0.014,0.016,0.018,0.022,0.023,0.048,0.061,0.097$.

\section{Symbolic Dynamics}

From Table 1 we verify that, as the coupling parameter $k$ grows and the two coupled maps begin to synchronize, the number of initial equal symbols in the $x$ and $y$ symbolic sequences, grows also and the distance previously 
defined, decreases. We have verified the same situation for others values of the $(\alpha, \beta)$ parameters. This gives numerical evidence of the quality of the synchronization between the two oscillators.

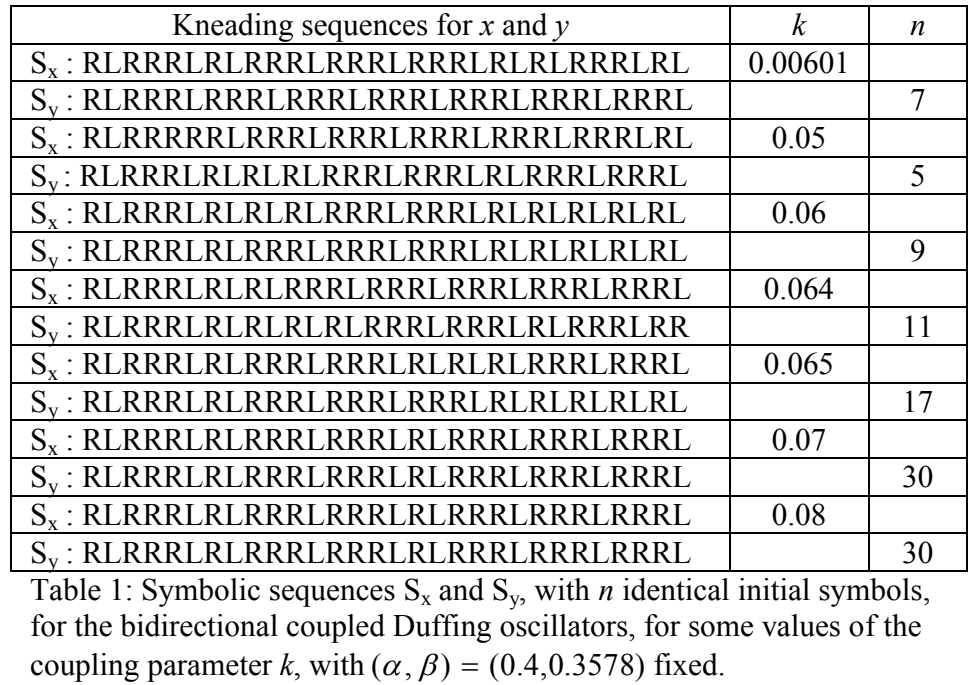

The number of symbols used in information transmission depends on the limitation of the channel capacity [13]. Only finitely precise synchronization is achievable when oscillators are joined by channel with limited capacity. On this basis, we established in Definition 2.1 and 2.2. finitely precise synchronization with symbolic dynamics.

\section{Conclusions}

Doing Poincaré sections in the system of two coupled Duffing oscillators, we obtained regions $U$ and $B$ where the Poincaré map behaves like a unimodal and bimodal map respectively. By a result from Milnor and Thurston [8] it is

known that every $m$-modal map $f: I=[a, b] \subset \mathrm{R} \rightarrow I$, with growth rate $s$ and

positive topological entropy $h_{\text {top }}(f)\left(\log s=h_{\text {top }}(f)\right)$ is topologically semiconjugated to a $p+1$ piecewise linear map $T$, with $p \leq m$, defined on the interval $J=[0,1]$, with slope $\pm s$ everywhere and $h_{\text {top }}(f)=h_{\text {top }}(T)=\log s$. Motivated by this fact, we study the synchronization of piecewise linear unimodal and bimodal maps and obtained the results in theorems 2.1 and 2.2 expressing the synchronization interval in terms of the topological entropy of the coupled maps. From the previous theorems we may also verify that the bidirectional coupled maps begin to synchronize at half the coupling 
parameter for the unidirectional case. We relate the synchronization with symbolic dynamics, namely, defining a distance between the kneading sequences and defining $n$-symbolic synchronization.

\section{References}

[1] S. Boccaletti, J. Kurths, G. Osipov, D. L. Valladares, C. S. Zhou, The synchronization of chaotic systems, Physics Reports 366: 1-101, 2002.

[2] A. Caneco, C. Grácio and J. L. Rocha, Symbolic dynamics and chaotic synchronization in coupled Duffing oscillators, J. Nonlinear Math. Phys. 15, 3: 102$111,2008$.

[3] A. Caneco, C. Grácio and J. L. Rocha, Kneading theory analysis of the Duffing equation, Chaos Solit. Fract. 42, (3): 1529-1538, 2009.

[4] A. Caneco, C. Grácio and J. L. Rocha, Topological entropy for the synchronization of piecewise linear and monotone maps. Coupled Duffing oscillators, Int. J. Bifurcation and Chaos, 19 (11): 3855-3868, 2009.

[5] A. P. Kurian and S. Puthusserypady, Secure digital communication using chaotic symbolic dynamics, Turk J. Engin 14 (1): 195-207, 2006.

[6] J. P. Lampreia and J. Sousa Ramos, Symbolic Dynamics for Bimodal Maps, Portugaliae Math. 54(1): 1-18, 1997.

[7] M. M. Ramos, C. C. Ramos, R. Severino and J. S. Ramos, Topological invariants of a chaotic pendulum, Int. J. Pure Appl. Math. 10 (2): 209-226, 2004.

[8] J. Milnor and W. Thurston, On iterated maps of the interval I and II, Lect. Notes inMath, 1342, Springer-Verlag: 465-563, 1988.

[9] L. M. Pecora and T. L. Carroll, Synchronization in chaotic systems, Phys. Rev. Lett. 64, 821-824, 1990.

[10] L. M. Pecora and T. L. Carroll, Driving systems with chaotic signals, Phys. Rev. A 44: 2374-2383, 1991.

[11] A. Pikovsky, M. Rosenblum and J. Kurths, Synchronization:a universal concept in nonlinear sciences, Cambridge University Press, 2001.

[12] U. E. Vincent and A. Kenfack, Synchronization and bifurcation structures in coupled periodically forced non-identical Duffing oscillators, Phys. Scr. 77: 045005 (7pp), 2088.

[13] X. Wang, M. Wang and Z. Liu, Chaos synchronization basing on symbolic dynamics with nongenerating partition, Chaos 19: 023108, 2009. 